\title{
STUDY OF THE RADIATION INDUCED EFFECTS IN THE LHCb VERTEX LOCATOR
}

\author{
TOMAsz SzumLak \\ on behalf of the LHCb VELO Group \\ AGH University of Science and Technology \\ al. Mickiewicza 30, 30-059 Kraków, Poland
}

(Received November 18, 2015)

\begin{abstract}
$\mathrm{LHCb}$ is a dedicated heavy-flavour physics experiment at the Large Hadron Collider at CERN. The VErtex LOcator (VELO) is a critical part of a LHCb tracking system, enabling the full topological reconstruction of beauty and charm mesons' decays and providing essential input for the High Level Trigger (HLT) system used by the experiment to select events. The VELO comprises 42 modules made of two $n^{+}$-on- $n 300 \mu \mathrm{m}$ thick halfdisc silicon sensors with $R$ - and $\Phi$-measuring micro-strips, arranged in two retractable halves, operating only about $8 \mathrm{~mm}$ from the proton beams. In these paper, selected aspects of the VELO performance during the Run 1 data-taking period is shortly summarised along with the radiation damage studies. The track finding efficiency is typically greater than $98 \%$. An impact parameter resolution of less than $35 \mu \mathrm{m}$ is achieved for particles with transverse momentum greater than $1 \mathrm{GeV} / c$. An overview of all important performance parameters will be given. The VELO sensors have received a large and non-uniform radiation dose of up to $1.2 \times 10^{14} 1 \mathrm{MeV}$ neutron equivalent $\mathrm{cm}^{-2}$. Silicon-type inversion has been observed in regions close to the interaction point.
\end{abstract}

DOI:10.5506/APhysPolB.47.441

\section{Introduction}

The LHCb Vertex Locator (VELO) [1] is a crucial part of the LHCb [2] tracking system able to perform standalone tracking procedure that is vital for precise reconstruction of the primary and secondary vertices what, in turn, has a direct impact on measurement of the lifetime of decaying particles and determine the geometrical impact parameter. It makes a vast contribution to the High Level Trigger (HLT). The information provided by the VELO is used to suppress the background, since the displaced vertices in 
a given event make an excellent indicator of the presence of heavy flavoured mesons. The VELO is positioned around the interaction point and its main purpose is to provide precise track reconstruction of charged particles (see figure 1). The remainder of this paper is split into two sections describing the VELO performance and selected topics regarding the radiation damage induced by the proton-proton collisions.

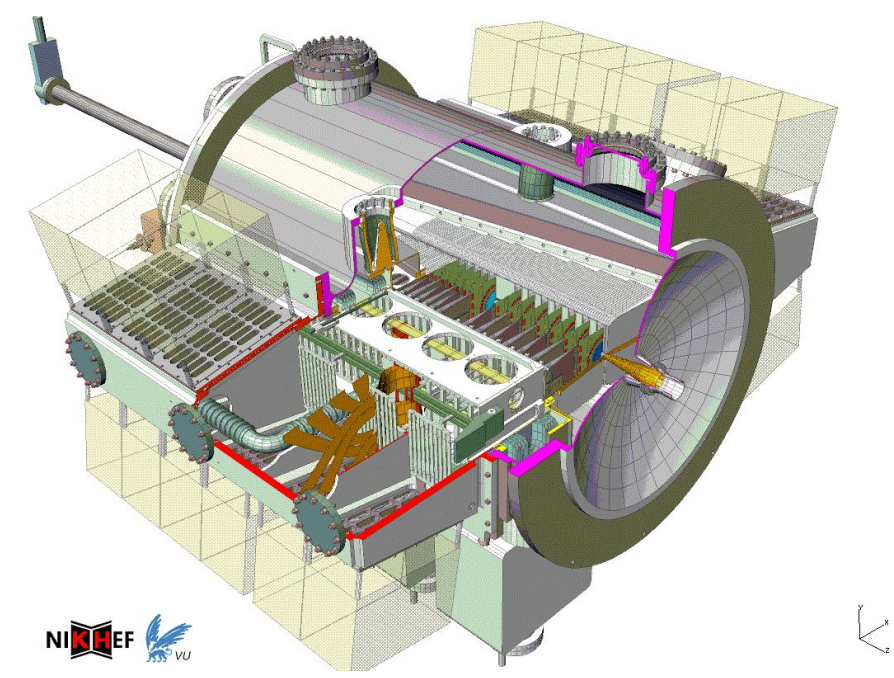

Fig. 1. A perspective view of the VELO with its vacuum tank and modules.

\section{VELO performance}

In this chapter, selected quantities, that should be viewed as figures of merit for the VELO performance, are presented [3].

\subsection{Signal and noise measurement}

In order to maintain excellent performance of the VELO throughout the collision data-taking period, the signal-to-noise $(\mathrm{S} / \mathrm{N})$ ratio must be constantly monitored. To evaluate the $\mathrm{S} / \mathrm{N}$ ratio, clusters assigned to reconstructed tracks were used. The procedure of determining the $\mathrm{S} / \mathrm{N}$ goes as follows: first, for each sensor, an ADC (Analogue to Digital Conversion) distribution was created. Next, a convoluted Landau and Gaussian model was fitted and the most probably value (MPV) extracted. The obtained MPV is almost uniform for all VELO sensors and close to 37 ADC counts (see figure 2). The noise values measured in $R$ and $\Phi$ sensors [1] are very similar and close to $2 \mathrm{ADC}$ counts (see figure 3 ). The average signal-to-noise value is determined to be around 19 and 21 for $R$ - and $\Phi$-type sensors respectively. 


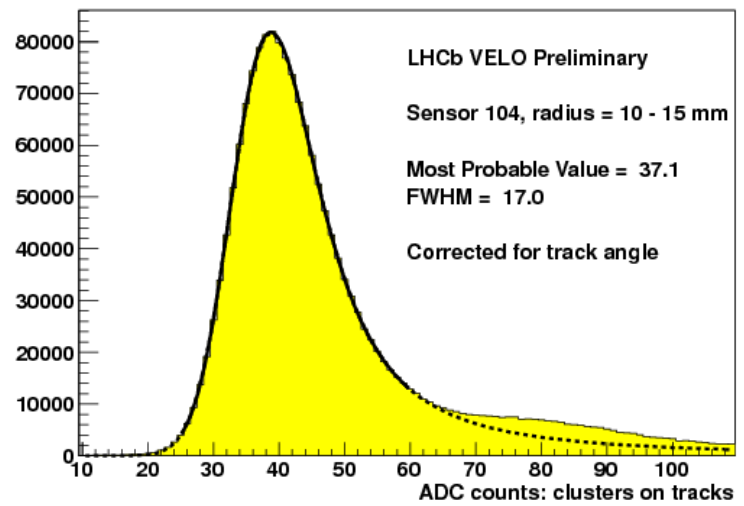

Fig. 2. Landau convolved with a Gaussian to fit to ADC count distributions for clusters on tracks in a selected VELO sensor. The peak and the full width at half maximum values are shown on the plot. The data has been corrected for the track angle to make every entry equivalent to a track crossing 300 microns of Si.

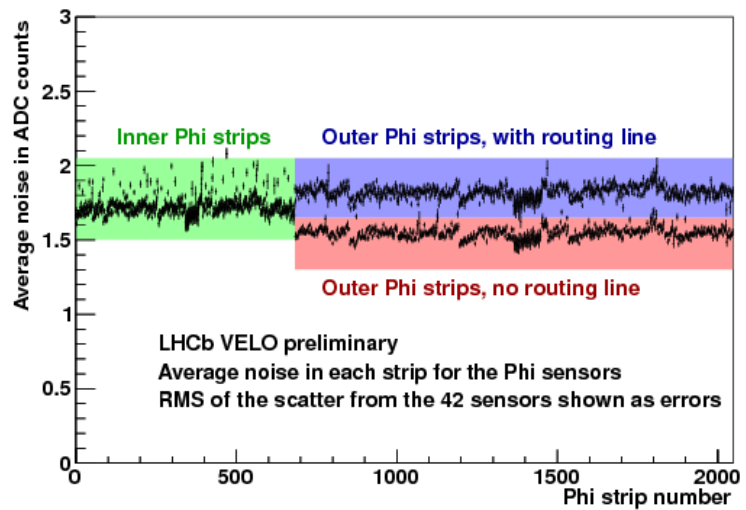

Fig. 3. Noise in the Phi sensors by strip number averaged over all VELO sensors, with the RMS of the distribution as the error. The higher points with more RMS are the first channels in each link. The three coloured regions indicate the strips in the inner region.

\subsection{VELO spatial resolution}

At the beginning of each new LHC injection cycle, the VELO halves need to be retracted for the safety reason. It makes necessary to re-evaluate the spatial alignment constants on fill-by-fill basis. Also, any time-dependent variation in the alignment must be well-understood in order to keep the highest quality of physics data. The VELO alignment procedure is based on measuring residuals between track intercept point and the reconstructed 
cluster position. From the point of view of the regular operation, the critical part of this procedure is inter-half alignment, which is determined using the displacement between primary vertices (PVs) reconstructed separately by each VELO half [4]. The module and sensor alignment is known to be around $4 \mu \mathrm{m}$ whilst the half-to-half alignment is shown to be stable over time to better than $5 \mu \mathrm{m}$. The single hit resolution is estimated using unbiased tracks residuals. In order to measure the unbiased hit resolution for a given sensor, this sensor must be excluded from the Kalman-based fitting procedure. It has been shown that the spatial resolution depends, in non-trivial way, on the sensor strip pitch and projected angle of a track that is used to calculate the residual. For the optimal angle and smallest pitch, the resolution is better than $4 \mu \mathrm{m}$, and is the best one for vertex detectors at the LHC. The optimal projected angle is found to be close to 8 degrees.

\subsection{Primary vertex and geometrical impact parameter resolution}

For the primary vertex (PV) resolution, a specially designed data driven algorithm has been used. For each event, the reconstructed tracks are split randomly into two equally sized sets. Next, each set is used separately to fit a PV and the distance between the two PVs is taken as a measure of the PV resolution. The obtained resolution depends strongly on the number of tracks used in the fitting procedure. For 25 tracks per each vertex, the respective PV resolutions are as follows: $\sigma_{x, y}=13.0 \mu \mathrm{m}$ and $\sigma_{z}=69.0 \mu \mathrm{m}$. The impact parameter (IP) is the key discriminating variables that is being used in the LHCb HLT trigger to select the long-lived heavy charm and beauty mesons. It is defined as the distance of the closest approach of a given track to the PV. The PV resolution depends on both the single hit resolution and multiple scattering. For the high momentum particles, the IP measured by the VELO is close to $12 \mu \mathrm{m}$.

\section{Radiation damage}

The VELO sensors are exposed to a very harsh hadronic environment of the proton-proton collisions with the first active strip positioned only $8.2 \mathrm{~mm}$ from the beam. On top of that, their particular orientation (perpendicular to the beam) is the source of highly non-uniform radiation damage. It is estimated that the maximum fluence seen by the VELO at the inner-most regions is close to $4.5 \times 10^{14} 1 \mathrm{MeV} \mathrm{n} \mathrm{neq} \mathrm{cm}^{-2}$. Since the performance of the VELO is crucial for the entire LHCb physics programme, the radiation damage to its sensors is of great concern and it has been monitored carefully using a number of independent methods. Especially, the increase in bulk current and effective depletion voltage are of particular interest as two figures of merit for estimating the radiation damage effects. The former can 
be estimated by monitoring the leakage current measured as a function of applied bias voltage. This quantity is expected to increase approximately linearly with the fluence. The latter is estimated by measuring the bias voltage that gives charge collection efficiency of $80 \%$. The increase of the bulk current with the fluence is in very good agreement with the predictions of the Hamburg model and it is measured to be close to $1.9 \mu \mathrm{A} \mathrm{pb}^{-1}$ [5]. The reverse bias leakage current in silicon sensors varies almost linearly with the delivered fluence, when full depletion voltage is reached. Increase of sensor current, observed during the collision data taking, is a direct indication of radiation damage in silicon. The total leakage current for a particular sensor is a combination of bulk and surface components. The surface part of the total measured current decreases with fluence, while the bulk contribution is dominant after severe irradiation and shows linear dependence on fluence.

The bulk component of the currents varies exponentially with temperature (see figure 4). Measurements of the reverse current as a function of temperature (so-called $I / T$ scan) allow to identify the bulk contribution and to extract the effective band gap, $E_{\mathrm{g}}$, of silicon. The $I / T$ scans taken after the Run 1 have been finished, for all the sensors, are presented in figure 4 . Points are fitted with an exponential function and the obtained value (based on dataset of $2.7 \mathrm{pb}^{-1}$ of collision data) is $E_{\mathrm{g}}=1.16 \pm 0.06 \mathrm{eV}$, which is consistent with theoretical predictions [6].

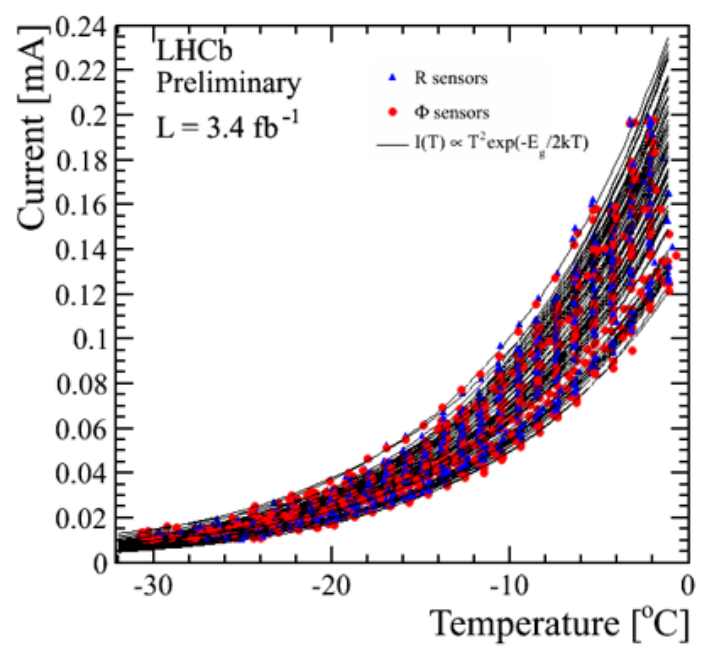

Fig. 4. The leakage current versus temperature for all VELO sensors at a bias voltage of $150 \mathrm{~V}$.

This research was supported in part by PL-Grid Infrastructure which acts as the TIER-2 computing centre for the LHCb. 


\section{REFERENCES}

[1] LHCb Collaboration, LHCb Vertex Locator Technical Design Report, CERN/LHCC 2001-0011 (2001).

[2] LHCb Collaboration, JINST 3, S08005 (2008).

[3] LHCb VELO Group, JINST 9, P09007 (2014).

[4] S. Borghi et al., Nucl. Instrum. Methods A 618, 108 (2010).

[5] LHCb VELO Group, JINST 8, P08002 (2013).

[6] A. Chilingarov, JINST 8, P10003 (2013). 